\title{
Characterization of a Wheat Heme Oxygenase-1 Gene and Its Responses to Different Abiotic Stresses
}

\author{
Dao-kun Xu ${ }^{1}$, Qi-jiang Jin ${ }^{1}$, Yan-jie Xie ${ }^{1}$, Ya-hui Liu ${ }^{1}$, Yu-ting Lin ${ }^{1}$, Wen-biao Shen ${ }^{1}{ }^{*}$ and \\ Yi-jun Zhou ${ }^{2, *}$
}

1 College of Life Sciences, Cooperative Demonstration Laboratory of Centrifuge Technique, Nanjing Agricultural University, Nanjing 210095, China; E-Mails: 2009116134@njau.edu.cn (D.X.); 2010216030@njau.edu.cn (Q.J.); 2008216036@njau.edu.cn (Y.X.); webmasterone@ sina.com (Y.-h.L.) 2010116133@njau.edu.cn (Y.-t.L.)

2 Institute of Plant Protection, Jiangsu Academy of Agricultural Sciences, Nanjing 210014, Jiangsu, China

* Authors to whom correspondence should be addressed; E-Mails: wbshenh@ @jau.edu.cn (W.S.); yjzhou@jaas.ac.cn (Y.Z.); Tel./Fax: +86-25-8439-6542 (W.S.); +86-25-8439-0391(Y.Z.).

Received: 4 September 2011; in revised form: 31 October 2011 / Accepted: 1 November 2011 / Published: 8 November 2011

\begin{abstract}
In animals and recently in plants, heme oxygenase-1 (HO1) has been found to confer protection against a variety of oxidant-induced cell and tissue injuries. In this study, a wheat (Triticum aestivum) $\mathrm{HO} 1$ gene $\mathrm{TaHOl}$ was cloned and sequenced. It encodes a polypeptide of $31.7 \mathrm{kD}$ with a putative $N$-terminal plastid transit peptide. The amino acid sequence of TaHO1 was found to be $78 \%$ similar to that of maize HO1. Phylogenetic analysis revealed that $\mathrm{TaHO} 1$ clusters together with the HO1-like sequences in plants. The purified recombinant TaHO1 protein expressed in Escherichia coli was active in the conversion of heme to biliverdin IXa (BV), and showed that the $V_{\max }$ was $8.8 \mathrm{U} \cdot \mathrm{mg}^{-1}$ protein with an apparent $K_{m}$ value for hemin of $3.04 \mu \mathrm{M}$. The optimum Tm and $\mathrm{pH}$ were $35{ }^{\circ} \mathrm{C}$ and 7.4, respectively. The result of subcellular localization of TaHO1 showed that the putative transit peptide was sufficient for green fluorescent protein (GFP) to localize in chloroplast and implied that TaHO1 gene product is at least localized in the chloroplast. Moreover, we found that $\mathrm{TaHOI}$ mRNA could be differentially induced by the well-known nitric oxide (NO) donor sodium nitroprusside (SNP), gibberellin acid (GA), abscisic acid (ABA), hydrogen peroxide $\left(\mathrm{H}_{2} \mathrm{O}_{2}\right)$ and $\mathrm{NaCl}$ treatments. Therefore, the results suggested that $\mathrm{TaHOl}$ might play an important role in abiotic stress responses.
\end{abstract}


Keywords: abiotic stress responses; carbon monoxide; heme oxygenase-1; prokaryotic expression in E. coli; wheat

\section{Introduction}

A wide range of environmental stresses, such as salinity, drought, UV, and heavy metals exposure, are potentially harmful to plants. A common aspect to all of these adverse conditions is the enhanced production of reactive oxygen species (ROS) within several subcellular compartments of the plant cells [1]. In order to cope with above abiotic stresses, some antioxidative systems such as superoxide dismutase (SOD), catalase (CAT), and ascorbic acid peroxidase (APX) are proved responsible for the protection against toxic ROS [2]. Recently, another system, heme oxygenase-1 (HO1), which belonged to the inducible and major isoform of heme oxygenases (HOs; EC 1.14.99.3), gained more attention in both animals and plants because of its assumed responsibility for the detoxification of ROS and free radicals [3]. It was well established that $\mathrm{HO}$ could catalyze the oxidation of heme with molecular oxygen, which results in the formation of biliverdin-IX $\alpha(\mathrm{BV})$, carbon monoxide (CO) and free iron [4-7]. In mammals, BV and its reduced derivatives, bilirubin (BR), have strong antioxidant properties both in vitro and in vivo [4,8], thus resulting in the speculation that $\mathrm{HO} 1$ might have a protective role against tissue injury [9].

In Arabidopsis, HOs include a small gene family with four members in total which can be categorized into two subfamilies, $\mathrm{HO} 1$ and $\mathrm{HO} 2$ [10,11]. It is known that $\mathrm{HO} 1$ family includes three isoforms: $\mathrm{HY} 1, \mathrm{HO} 3$, and $\mathrm{HO} 4$, while the $\mathrm{HO} 2$ family only has one member: $\mathrm{HO} 2$. All the four members of the $\mathrm{HO}$ family are transcriptionally active with substantially overlapping patterns of expression. Some evidence also showed that HO1 is clearly the most highly expressed, followed by $\mathrm{HO} 2$, while both $\mathrm{HO} 3$ and $\mathrm{HO} 4$ expressed at low levels [10]. In other plants, HO1 has been proved to be an inducible enzyme and can be induced by multiple stimuli and various abiotic stresses, including its own substrate heme [12], heavy metals [13,14], glutathione depletion [15], UV radiation [16], salinity stress [17-19], and $\mathrm{H}_{2} \mathrm{O}_{2}$ [20]. These responses were thus thought to be a cellular defense mechanism against various stresses-triggered oxidative damage and also exhibit hormone-like bioactivity [3,21].

Until now, the researches on plant HO1 genes have only focused on a few plant species such as Arabidopsis (AtHO1, also called HY1) [22], rice (OsHO-1, also called SE5) [23], alfalfa (MsHO1) [12], and Chinese cabbage (BrHOl) [24], etc. Wheat (Triticum aestivum) is one of the most important crops worldwide. To date, the characterization of wheat HO1 gene remained unknown because of its limited genomic information [19]. However, analysis of wheat expressed sequence tags (ESTs) generated from various tissues during plant development or upon different stress conditions gives insights into the transcribed portions of the genome. In this test, to get first insight into the role of wheat HO1, we reported on molecular cloning and characterization of a HO1 cDNA from wheat (named as TaHOl). Sequence analyses of the deduced amino acids of its encoded protein were analyzed and their phylogenetic relationships were compared with other HOs from various plant species. Furthermore, subcellular localization of TaHO1 protein was surveyed. We further purified recombinant mature TaHO1 protein expressed from an Escherichia coli expression system and detected its HO enzyme 
activity successfully, then characterized this protein in terms of spectroscopic and catalytic properties. The expression patterns of $\mathrm{TaHOl}$ by real-time RT-PCR were investigated in various wheat seedling tissues under the normal growth conditions and with different exogenous chemicals and salinity stress. Therefore, the characterization of $\mathrm{TaHOl}$ will provide insight into the physiological processes of stress responses in wheat plants.

\section{Results and Discussion}

\subsection{Identification and Cloning of a Wheat HOl Gene}

Abundant ESTs in public databases provide a source for the identification of new genes and for comparative analyses among different organisms. In this study, we searched the wheat EST database in NCBI with the maize $\mathrm{HOl}$ sequence. This search obtained several EST sequences with high homology to the query, and a putative wheat $\mathrm{HOl}$ gene was assembled by ORF analysis. One full length cDNA of 867 bp was amplified from wheat seedling leaves with primer pairs P1 designed based on this assembled $\mathrm{HOl}$. We noticed that the predicted protein product of its sequence comprises 288 animo acids which shared $99 \%$ identity with the deduced product of the assembled wheat $\mathrm{HOl}$ sequence. Furthermore, we discovered that this sequence was identical to the maize $\mathrm{HOl}$ and designated as TaHO1. For example, the nucleotide sequence showed the highest similarity (60\%) to that of the maize HOl gene, within the predicted coding region and the putative translated product was $78 \%$ identical. The predicted molecular mass of TaHO1 was $31.7 \mathrm{kD}$ and its theoretical isoelectric point was 6.41.The sequence of this cDNA was deposited in GenBank with the accession ID HM014348.

Like that of the Arabidopsis counterpart HY1 [22], computer analysis by the ChloroP program predicted that TaHO1 might also localize into the chloroplast, and its predicted chloroplast transit peptide cleavage site is most likely positioned between amino acids 62 and 63 (Figure 1). The alignment of the amino acid sequences of wheat and other $\mathrm{HO} 1$ further illustrated that a total of 52.7\% of residues is conserved, and almost all amino acids are conserved in the HO signature sequence (QAFICHFYNI/V) which corresponds to Q199-V208 in TaHO1 (Figure 1, boxed). The signature sequence was also conserved in mammalian HO1 sequences [5]. Interestingly, Arabidopsis HY1 contains four histidine residues and all of them are conserved in other plant HO1. In fact, although the amino acid sequences of these plant HO1 proteins are not closely related to mammalian HO sequences [22], His-132 in rat HO-1, which is thought to play a structural role in stabilizing the HO protein [25], is maintained in TaHO1 as His 204. The proximal heme-binding ligand of human HO1, His25 [26], is also conserved in TaHO1 as His95. Previous report demonstrated that in the first step of the reaction of HO, heme bound to an amino acid residue of the HO protein through the fifth co-ordination portion of the iron atom to form an enzyme-substrate complex, and that the absorption spectrum of the complex formed closely resembled that of hemoglobin and myoglobin [27]. Therefore, some of these histidine residues, conserved in those sequences, are probably important for heme binding. Together, all these data clearly suggested that this cDNA encodes HO. 
Figure 1. The wheat $\mathrm{HOl}$ gene. Alignment of TaHO1 with other plant HO1s. Dark shading with white letters and gray shading with black letters reveal $100 \%$ and $75 \%$ sequence conservation, respectively. Database accession numbers are ADG56719 for TaHO1 (Triticum aestivum), AB021858 for AtHO1 (Arabidopsis thaliana), GU390397 for BnHO1 (Brassica napus), AF320024 for GmHO1 (Glycine max), AF320028 for LeHO1 (Lycopersicon esculentum), EU781632 for OsHO1 (Oryza sativa), AF276228 for PsHO1 (Pisum sativum), and EU962994 for ZmHO1 (Zea mays), respectively. Solid frame means signature sequence. Also, the arrow identified the predicated cleavage site between the transit peptide and mature protein. The conserved histidine residue involved in heme-iron binding and catalysis was shown by a white diamond. The conserved histidine residue for protein stability was illustrated by a reverse triangle.

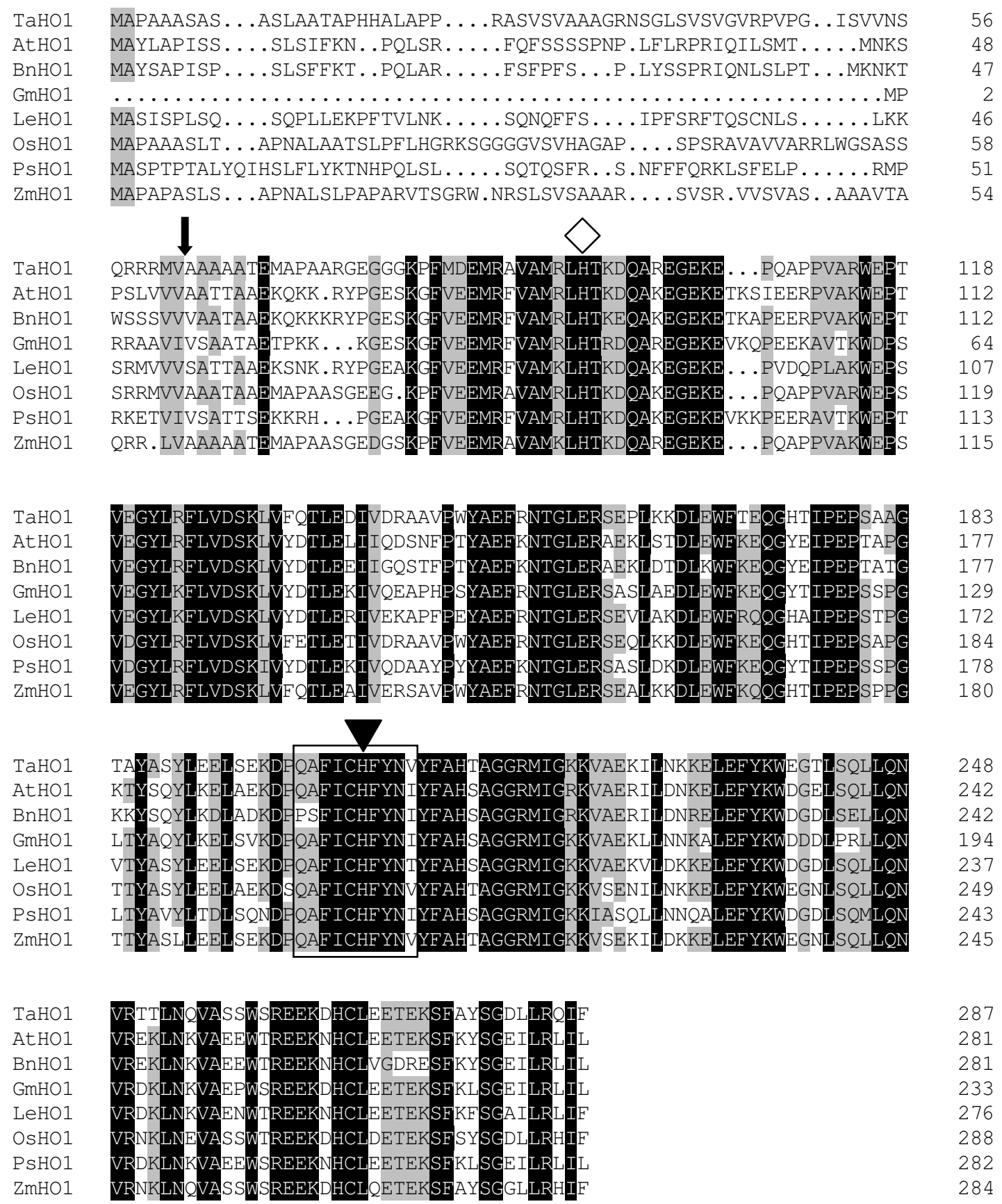


Figure 2. The phylogenetic relationships of mature HO1/2 isoforms in plants. The protein sequence for the mature region of TaHO1 was aligned with mature sequences of previously identified plant HO proteins. Besides shown in Figure 1, other database accession numbers are as follows: Brassica campestris (BrHO1, HQ690823), Arabidopsis thaliana (AtHO2, AAD22109; AtHO3, NP177130; AtHO4, AAK63007), Glycine max (GmHO3, AAK63009), Medicago sativa (MsHO1, ADK12637; MsHO2, HQ652868), Cucmis sativus (CsHO1, ADO08223), Nicotiana tabacum (NtHO1, HQ690822), Lycopersicon esculentum (LeHO2, AF320029.1), Oryza sativa (OsHO2, BM293897.1), Pinus taeda (PtHO1, AAK63014), Sorghum bicolor (SbHO2, AAK63011) and Gossypium hirsutum (GhHO2, EU373020). Predicted $N$-terminal chloroplast transit peptide domains were removed based on predictions using ChloroP. Sequences were aligned by CLUSTALW and analyzed using the MEGA 4.1. Construction was carried out by the parsimony method with 1000 bootstrap replicates and a consensus tree was generated.

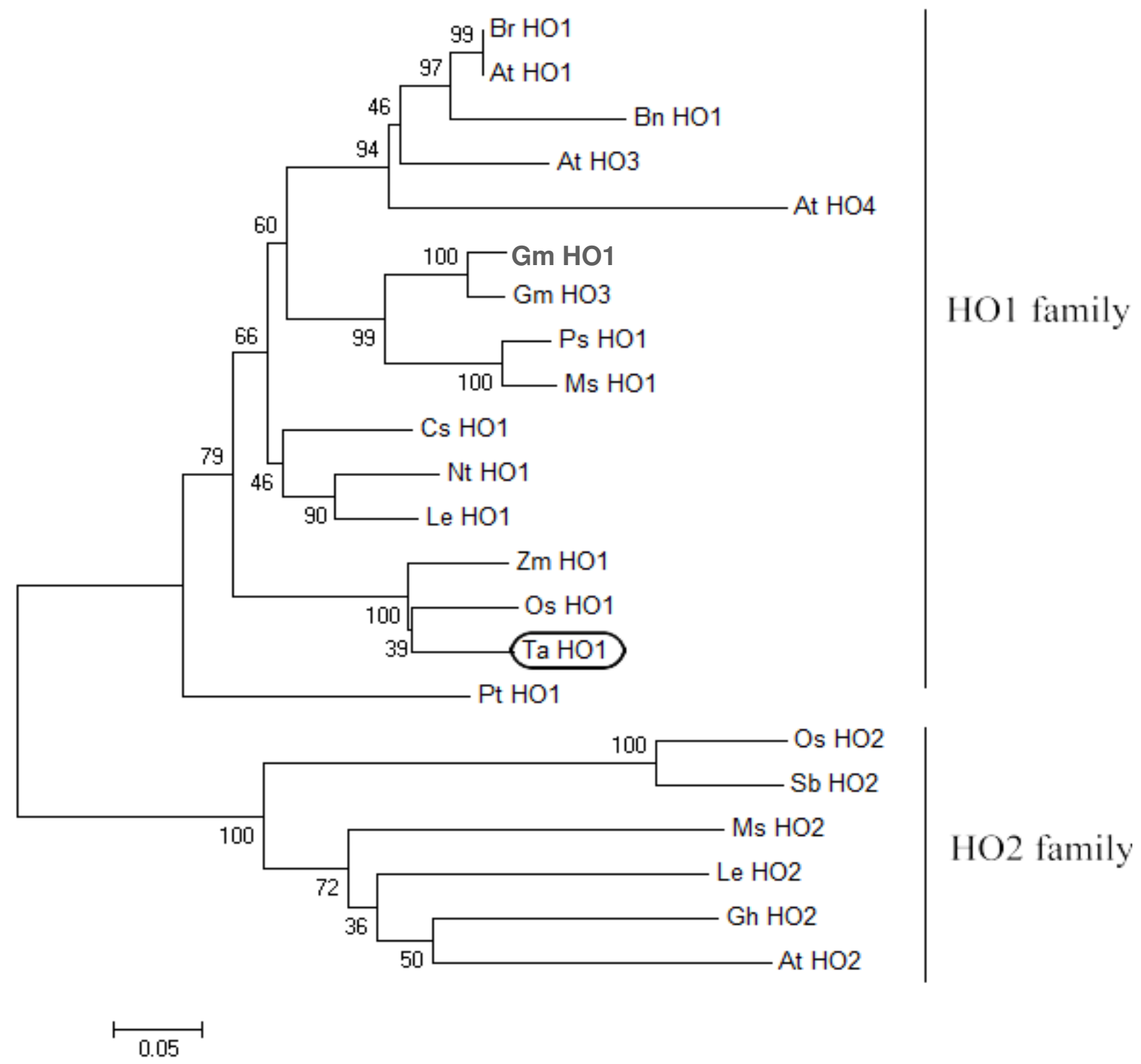




\subsection{Phylogenetic Analysis of TaHO1}

Other plant HOs discovered in GenBank were used for phylogenetic analysis to identify the evolutionary relationships among TaHO1 and other HOs (Figure 2). The $N$-terminal transit peptides of plant HOs were neglected in the analysis because this region was highly variable and not subject to the same evolutionary constraints as the regions encoding the mature protein. The phylogenetic tree showed that the plant HOs can be separated into two main divisions, HO1-like and HO2-like sequences. The TaHO1 was clearly grouped with the HO1-like sequences supporting its designation as TaHO1. Normally, plant HO1s group into a number of families based upon established taxonomic divisions. Consistent with this, $\mathrm{TaHOl}$ clearly groups with other sequences from the Poaceae, such as Zea mays and Oryza sativa (especially). Additionally, we found several HO1-like sequences existing in each species such as Arabidopsis (HO1, HO3, and HO4) while only one HO2-like sequence appeared. This indicates that the divergence of the two isoforms occurred before the speciation of these plants, and these HO1s might be a result of gene duplication of an ancestral copy of HO1 following the speciation.

Figure 3. Expression and analysis of the recombinant His-tagged mTaHO1 protein in E. coli. (A) Expressed proteins were induced with IPTG, $30 \mu \mathrm{g}$ protein/well. Lane M, Marker proteins; lane 1, protein from IPTG-induced bacteria for $12 \mathrm{~h}$ harboring pET-28a(+) alone as control; lane 2,3,5,7, and 9, proteins from uninduced bacteria for 0,1 , 3, 6 and $12 \mathrm{~h}$, respectively; lane 4,6,8 and 10, proteins from IPTG-induced bacteria for 1, 3, 6 and 12 h, respectively; (B) Expressed protein purified by Ni-affinity chromatography and its gel blot analysis, $60 \mu \mathrm{g}$ protein/well. Lane M, Marker proteins; lane 1, purified protein; lane 2, immunoblot of purified protein developed with the polyclonal antiserum against the mature wheat HO-1 (mTaHO1) with a molecular mass of $31 \mathrm{kDa}$.

A

B

$\begin{array}{llllllllllll}\mathrm{kD} & \mathrm{M} & 1 & 2 & 3 & 4 & 5 & 6 & 7 & 8 & 9 & 10\end{array}$

97.2

66.4

B

44.3

29.0

20.1

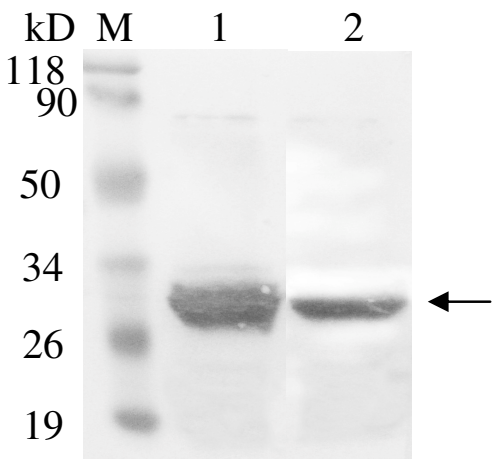

\subsection{Expression and Purification of TaHOI}

To confirm that TaHO1 encodes a $\mathrm{HO}$ and to further characterize its properties, we expressed the mature TaHO1 (mTaHO1) (without the predicted signal peptides) as the $N$-terminal His-tagged recombinant protein in $E$. coli by using the pET-28a(+) vector. The mature recombinant TaHO1 induced by IPTG was expressed as a soluble protein with the intensity in a time-dependent mode and formed a $31 \mathrm{kD}$ band (Figure $3 \mathrm{~A}$, lane 4,6,8,10), approximately corresponding to the molecular weight 
of the mTaHO1 protein $(25.6 \mathrm{kD})$ plus that of $6 \times$ his-tag $(0.8 \mathrm{kD})$ and the translated vector sequence $(4 \mathrm{kD})$. Whereas, E. coli. cells transformed with the empty vector did not contain proteins in this mass region (Figure 3A, lane 1). Furthermore, the purification of TaHO1 by Ni-affinity chromatography yielded a protein that formed a similar band on SDS-PAGE (Figure 3B, lane 1) and exhibited immunoreactivity with its specific antibody (Figure 3B, lane 2).

\subsection{Biochemical Activity of the Mature TaHOI Protein Expressed in Escherichia coli}

Furthermore, the purified recombinant protein was tested for heme ring cleavage activity by following the conversion of heme to BV spectrophotometrically. As shown in (Figure 4A), TaHO1 showed similar catalytic characteristics in heme cleavage as previously characterized HY1 in Arabidopsis [28], and BrHO1 in Chinese cabbage [24]. The enzyme first forms a complex with heme, which can be monitored photometrically and results in a maximum absorbance of the heme-TaHO1 complex at $405 \mathrm{~nm}$ (Figure 4A). Over a period of $30 \mathrm{~min}$, the reaction proceeded with a decrease in $405 \mathrm{~nm}$, since the complex was converted to a ferric biliverdin-HemT complex and subsequently to free biliverdin, as was apparent from the formation of a broad peak at approximate $660 \mathrm{~nm}$. These results indicated that this purified $\mathrm{TaHO} 1$ protein was able to degrade heme.

Figure 4. Biochemical characterization of purified recombinant mTaHO1. (A) Timedependent absorbance changes were determined during the mTaHO1 reaction with spectra between 360 and $700 \mathrm{~nm}$ taken at 1, 2, 5, 10, 20 and $30 \mathrm{~min}$ after the addition of NADPH. The black arrow indicates the degradation of hemin and the blue arrow stands for the generation of BV; (B) Michaelis-Menten plot of the mTaHO1 reaction for hemin concentrations of 1, 2, 5, 10 and $20 \mu \mathrm{M}$. Insert: Lineweaver-Burk plot of the same data; (C) Temperature (left) and $\mathrm{pH}$ (right) dependence of the $\mathrm{mTaHO} 1$ reaction. Data shown are the means $\pm \mathrm{SD}$ from three independent measurements.
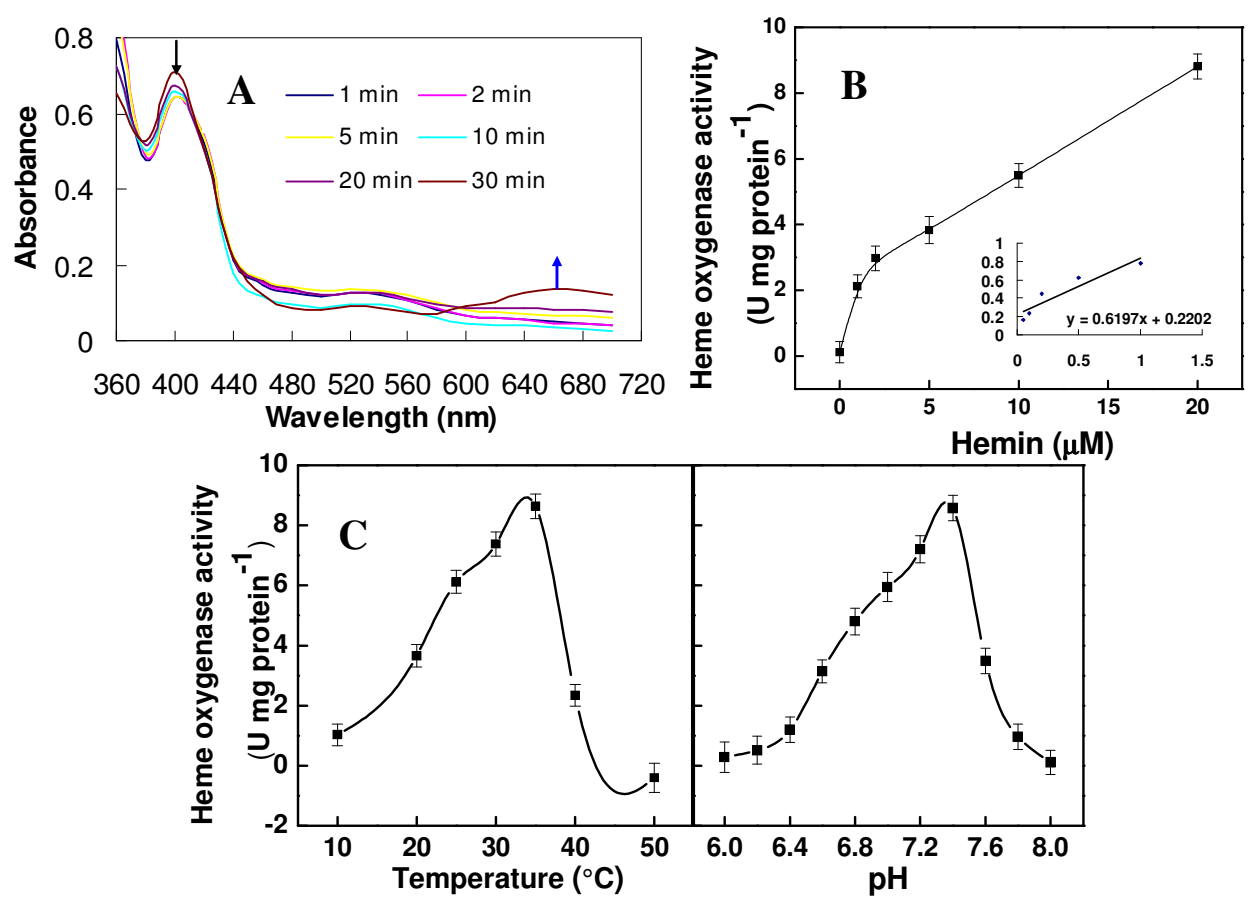
The plot of $\mathrm{HO}$ activity versus hemin concentration showed normal Michaelis-Menten kinetics (Figure 4B) and the kinetic constants were obtained from a Lineweaver-Burk plot (Figure 4B, insert). Under our experimental conditions, the $V_{\max }$ was $8.8 \mathrm{U} \cdot \mathrm{mg}^{-1}$ protein with an apparent $K_{\mathrm{m}}$ value for hemin of $3.04 \mu \mathrm{M}$. Comparable results were observed in previous analyses for human HO1 [29], Arabidopsis HY1 [28], alfalfa MsHO1 [12], and Chinese cabbage BrHO1 [24]. Additionally, the optimum Tm was $35{ }^{\circ} \mathrm{C}$ (Figure 4C), which is different from the values of $50{ }^{\circ} \mathrm{C}$ reported for recombinant Arabidopsis HY1 [28], and dropped sharply either side of this value. Meanwhile, $\mathrm{pH}$ dependence of the mTaHO1 reaction showed that the rate of the mTaHO1 reaction increased to a peak value at $\mathrm{pH} 7.4$ and declined thereafter.

\subsection{Subcellular Localization of Green Fluorescent Protein (GFP) Fusion Protein}

To demonstrate that predicted chloroplast targeting sequences of TaHO1 are able to sort the protein into chloroplast, a vector was made with a GFP fusion construct containing the predicted chloroplast transit peptide of TaHO1 upstream from GFP. After transfection of tobacco leave cells with EHA105 containing this construct, GFP was detected using confocal laser scanning microscopy in subcellular compartments with oval structure proven to be chloroplast as demonstrated by the overlap of red autofluorescence from the chlorophyll of the chloroplasts and the GFP fluorescence in it (Figure 5). The control construct without the putative transit peptide showed GFP fluorescence throughout the plasma membrane of tobacco leaves. This suggests the transit peptide of TaHO1 is functional and sufficient to transport TaHO1 to plastids, further implying that the $\mathrm{TaHOl}$ gene product in wheat plants is most likely localized in the plastids.

Figure 5. Subcellular localization of TaHO1 by transient expression of the fused fluorescent proteins. Microscopic images of GFP (A and D), chloroplast autofluorescence (B and E), and merge ( $\mathbf{C}$ and $\mathbf{F}$ ) from tobacco epidermal cells infected with Agrobacteria harboring the GFP constructs. GFP fused to TaHO1 transit peptide $(\mathbf{A}-\mathbf{C}$, scale bar $=20 \mu \mathrm{m})$ and GFP alone (negative control, D-F, scale bar $=25 \mu \mathrm{m})$. The photographs were taken in the blue channel (A and $\mathbf{D})$, red channel (B and $\mathbf{E})$, or their combination $(\mathbf{C}$ and $\mathbf{F})$.
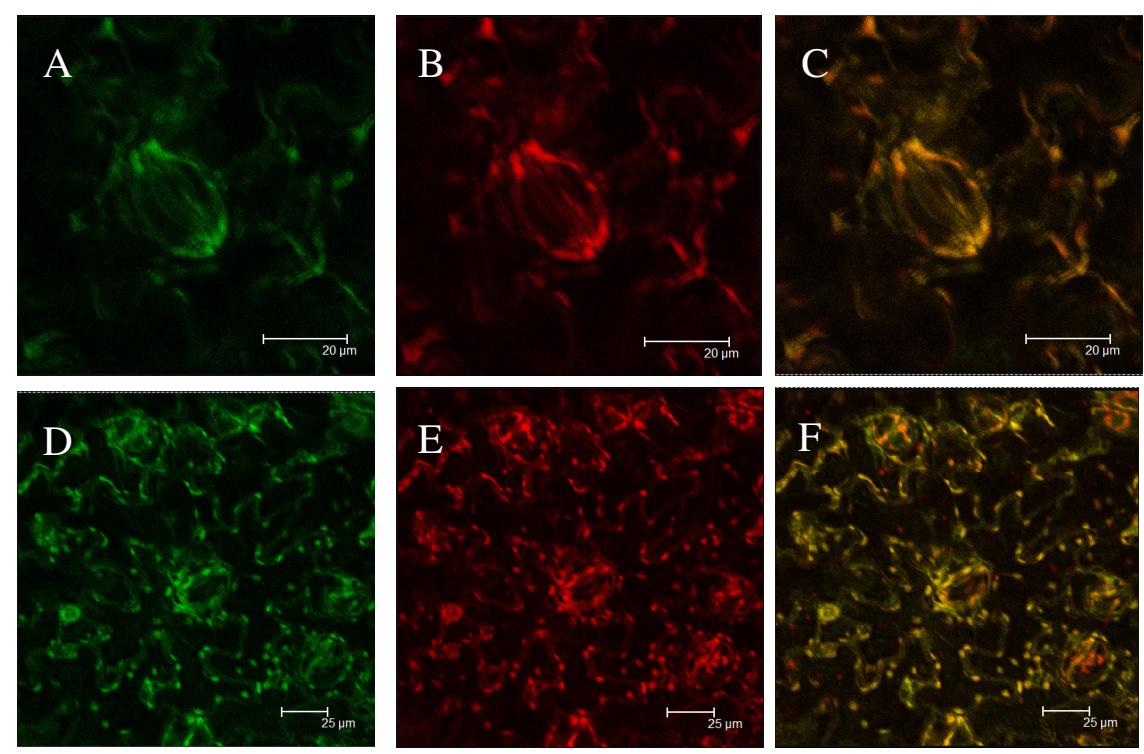


\subsection{Expression pattern Analysis of TaHO1 Protein}

The tissue-specific expression patterns of the TaHO1 protein in wheat seedlings were analyzed by gel blot analysis. The results of Figure 6 showed that TaHO1 protein levels were expressed in wheat leaves, stems, roots, and buds. It was also found that TaHO1 protein was more accumulated in stems and buds, but in roots with low levels (Figure 6). We also noticed that in germinating wheat seeds, two bands with a molecular mass of about $31 \mathrm{kD}$ appeared apparently, both of which were recognized by the rabbit polyclonal antibody against the TaHO1 protein. These suggested that another HO isoform as well as TaHO1, may play essential roles in wheat seed germination process.

Figure 6. TaHO1 protein levels in various wheat tissues. Young leaves (L), stems (S), roots (R), and buds (B) were collected from 14-d-old seedlings under the normal growth conditions. Meanwhile, germinating seeds (GS) were sampled after $3 \mathrm{~d}$ of germination. Then, TaHO1 protein levels were determined by protein gel blot analysis (A); Meanwhile, Coomassie Brilliant Blue-stained gels (B) were present to show that equal amounts of proteins were loaded. Densitometry was done by Quantity One software to quantify HO-1 protein level (C). Relative HO-1 protein level taking root sample (R) as $100 \%$.

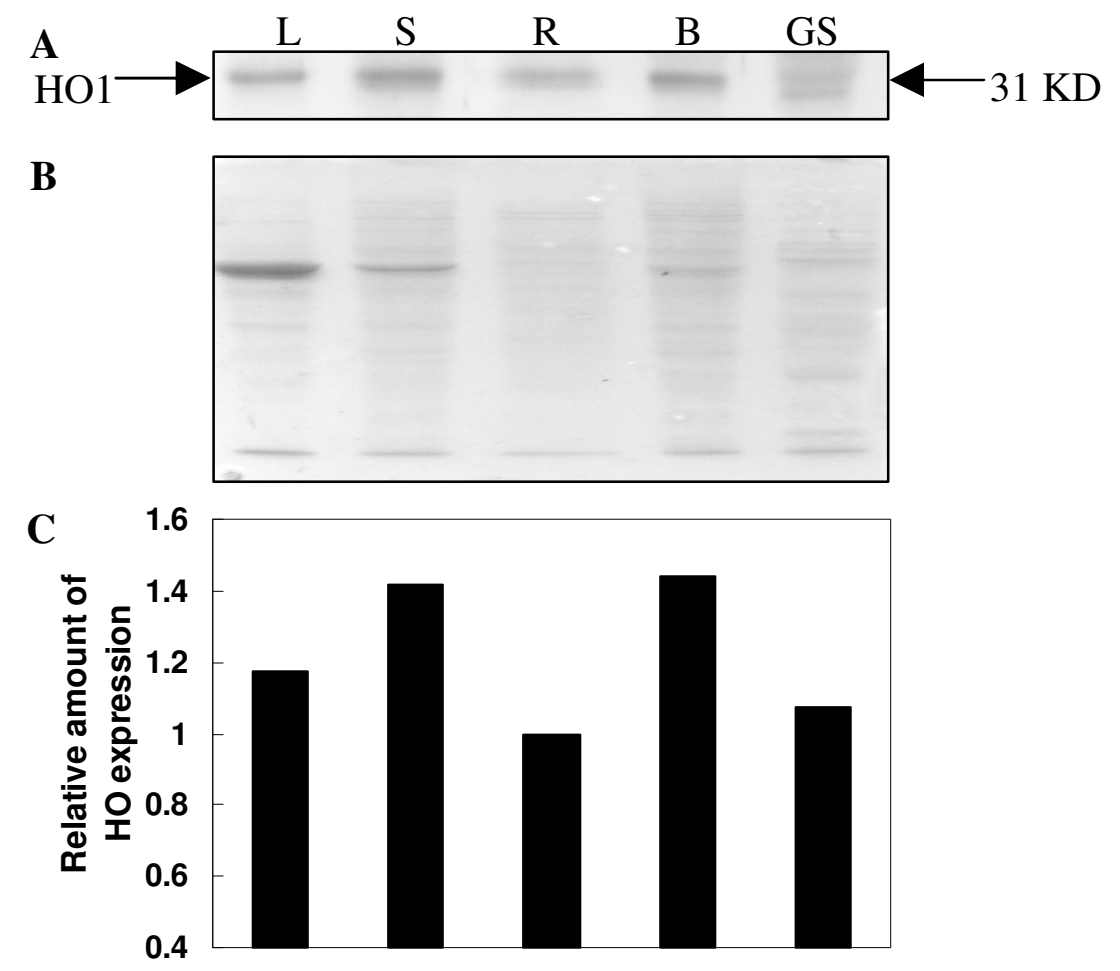

\subsection{Expression of TaHOI Gene in Responses to Different Exogenous Chemicals and Salinity Stress}

Recently, the HO1/CO system in plants has attracted more interest due to its physiological cytoprotective roles. For example, biological functions of HO-1 protein or corresponding genes are believed to be associated with a fundamental adaptive and defensive responses against osmotic and salinity stresses [17,18,30], UV irradiation [16], and heavy metal exposure [13,14]. In the subsequent test, the expression profiles of the $\mathrm{TaHOl}$ gene in wheat seedling leaves treated with different 
exogenous chemicals and $\mathrm{NaCl}$ were analyzed by real-time RT-PCR. Time-course analysis of gene expression showed that $\mathrm{TaHOl}$ transcripts could be differentially induced by SNP (sodium nitroprusside), $\mathrm{GA}, \mathrm{ABA}, \mathrm{H}_{2} \mathrm{O}_{2}$ and $\mathrm{NaCl}$ treatments (Figure 7). For example, TaHOl transcripts were up-regulated sharply by $\mathrm{NaCl}$ and SNP while gradually by $\mathrm{H}_{2} \mathrm{O}_{2}$ during the first $3 \mathrm{~h}$ treatment period then decreased except treated with $\mathrm{H}_{2} \mathrm{O}_{2}$. Meanwhile, a biphasic burst of $\mathrm{TaHOl}$ gene expression was appeared in GA treatment. The time course experiments illustrated that a fast increment of $\mathrm{TaHOI}$ transcripts appeared as early as $3 \mathrm{~h}$ after GA treatment, followed by a gradual decrease until reaching another peak until $12 \mathrm{~h}$.

Figure 7. Induction of $\mathrm{TaHOl}$ in response to $\mathrm{SNP}, \mathrm{GA}, \mathrm{ABA}, \mathrm{H}_{2} \mathrm{O}_{2}$, and $\mathrm{NaCl}$ treatments. Real-time RT-PCR was carried out from the total RNA isolated from the second leaves of wheat seedlings treated with $100 \mu \mathrm{M}$ SNP, $50 \mu \mathrm{M}$ GA, $100 \mu \mathrm{M} \mathrm{ABA}, 100 \mu \mathrm{M} \mathrm{H}_{2} \mathrm{O}_{2}$ or $100 \mathrm{mM} \mathrm{NaCl}$ at the indicated time points. Relative expression levels of $\mathrm{TaHOl}$ were presented relative to those of corresponding samples at $0 \mathrm{~h}$, respectively.

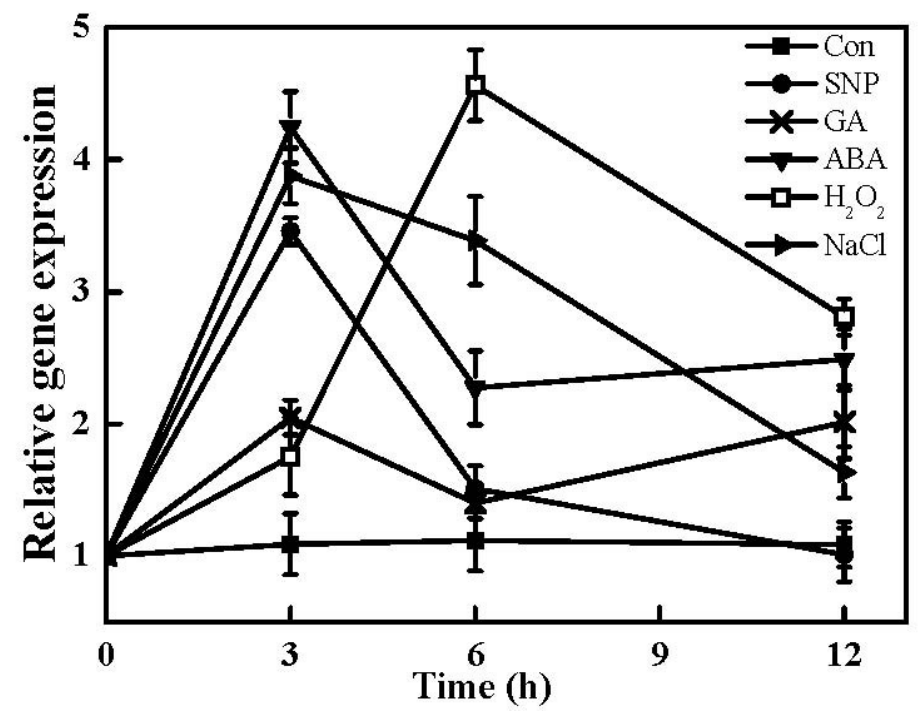

$\mathrm{NO}$ in the plant kingdoms has been suggested to act as a signal molecule mediating various responses to biotic and abiotic stresses, including salinity and osmotic stresses as well as UV irradiation [31]. Thus, the sharp enhancement of TaHOI transcripts induced by the NO donor SNP during the early stage of treatment indicated that $\mathrm{TaHOl}$ might be involved in plant tolerance against biotic and abiotic stresses through NO signaling pathway. Previously, studies showed that $\mathrm{HOl}$ gene from other plants could be induced by some agents or stimuli, including its own substrate heme [12], salinity stress [17,18], glutathione depletion [15], and $\mathrm{H}_{2} \mathrm{O}_{2}$ [20]. A common characteristic of these inducers is their ability to cause oxidative stress [3]. The results of Genevestigator DNA microarray database [32] showed that the HY1 mRNA was induced significantly in Arabidopsis seedlings upon osmotic and salinity stresses. Our recent study further showed that $H Y 1$ overexpression plant lines exhibited an enhanced salt tolerance [18]. Moreover, HO1 in soybean plants has been found to be induced significantly by UV-B irradiation [16] and salinity stress [33]. Although precisely how the expression of $\mathrm{HOl}$ is induced by these agents remains to be determined, in most cases, the induction has been confirmed to be associated with the alleviation of the oxidative stress. 
Thus, above findings of $\mathrm{TaHOl}$ suggested that the members of the $\mathrm{HO} 1$ isoform in plants are not only structurally similar, but also share a conserved role in plant responses against oxidative stresses [3]. Our results of responsiveness of $\mathrm{TaHOl}$ gene to $\mathrm{SNP}, \mathrm{GA}, \mathrm{ABA}, \mathrm{H}_{2} \mathrm{O}_{2}$ and $\mathrm{NaCl}$ also provide a possible link between the $\mathrm{HO} 1$ and the maintenance of cellular homeostasis.

\section{Experimental Section}

\subsection{Plant Materials, Growth Condition and Treatments}

All chemicals were obtained from Sigma (St Louis, MO, USA) unless stated otherwise. Commercially available wheat (Triticum aestivum, Yangmai 13) seeds were surface-sterilized with 5\% $\mathrm{NaClO}$ for $10 \mathrm{~min}$, rinsed extensively in distilled water and germinated for $1 \mathrm{~d}$ at $25^{\circ} \mathrm{C}$ in the darkness. Then, uniform buds were chosen and carefully transferred to the plastic chambers and cultured in modified Hoagland solution [17]. Wheat seedlings were grown in the illuminating incubator at $25 \pm 1{ }^{\circ} \mathrm{C}$, with a light intensity of $300 \mu \mathrm{mol} \cdot \mathrm{m}^{-2} \cdot \mathrm{s}^{-1}$ and $12 \mathrm{~h}$ photoperiod. The culture solution was renewed every other day until two fully expanded leaves appeared. Then, wheat seedlings were incubated in modified Hoagland solution containing $100 \mu \mathrm{M}$ sodium nitroprusside (SNP), the well-known nitric oxide (NO) donor, $50 \mu \mathrm{M}$ GA, $100 \mu \mathrm{M}$ ABA, $100 \mu \mathrm{M} \mathrm{H}_{2} \mathrm{O}_{2}$ or $100 \mathrm{mM} \mathrm{NaCl}$. The second fully expanded leaves were harvested at $0,3,6$ and $12 \mathrm{~h}$ of various treatments. For tissue-specific expression analysis, young leaves (L), stems (S), roots (R), and buds (B) were collected from 14-d-old seedlings under the normal growth conditions. Meanwhile, germinating seeds (GS) were sampled after $3 \mathrm{~d}$ of germination. All harvested samples were immediately used or frozen in liquid nitrogen, and then stored at $-80{ }^{\circ} \mathrm{C}$ until further analysis. Three different experiments were performed, with at least three replicated measurements for each parameter assayed.

\subsection{Cloning of $\mathrm{TaHOI}$}

Blast [34] searches were employed to search wheat ESTs in the dbEST databases that were homologous with the mRNA sequence of the maize full-length HOl gene (GenBank ID: NM_001199033). A putative wheat $\mathrm{HOl}$ gene sequence was assembled by comparative alignments of corresponding EST sequences. The specific sequences were used to design PCR primer pairs P1 (F: 5'-TCCGGATCCATGGCGCCCGCGGCAGC-3' and R: 5'-GTTAAGCTTTTAGGTGAATATCTGGCGTAG-3'), which would amplify the entire coding region of wheat $H O 1$ cDNA by RT-PCR.

Total RNA was extracted from wheat seedling leaves using Trizol reagent (Invitrogen, Gaithersburg, MD) according to the manufacturer's instructions. First-strand cDNAs were synthesized from $2 \mu \mathrm{g}$ total RNA (pre-treated with DNase I) with AMV reverse transcriptase (TaKaRa) according to the manufacturer's protocol. The PCR conditions for amplifying TaHO1 were as follows: 10 min at $94{ }^{\circ} \mathrm{C}$; 32 cycles of $30 \mathrm{~s}$ at $94{ }^{\circ} \mathrm{C}, 50 \mathrm{~s}$ at $50{ }^{\circ} \mathrm{C}, 1 \mathrm{~min}$ at $72{ }^{\circ} \mathrm{C}$; and a final extension for $10 \mathrm{~min}$ at $72{ }^{\circ} \mathrm{C}$. The PCR product was gel-purified and cloned into pMD-19T vector (TaKaRa) for sequencing (GenScript, Nanjing, China). 


\subsection{Multiple Sequence Alignment and Phylogenetic Analysis}

Alignment of deduced $\mathrm{TaHO} 1$ or other plant $\mathrm{HO}$ protein sequences were performed with DNAMAN 6.0.40 software. The phylogenetic tree of mature plant HO proteins was constructed with MEGA program (ver 4.1) by neighbor-joining (NJ) method. The parameters pairwise deletion and p-distance model were used. Bootstrap test of phylogeny was performed with 1000 replicates.

\subsection{Prokaryotic Expression and Purification of Recombinant His-Tagged TaHO1}

A mature TaHO1 (mTaHO1) without the predicted chloroplast transit peptide was subcloned into the Escherichia coli expression vector pET-28a (+) (Novagen) to produce pET-28a(+)-mTaHO1. mTaHO1 was amplified using the primer pairs P2 (F: 5'-AGGAGGGGATCCGCGGCGGCGGCGGCGAC3' and R: 5'-GTTAAGCTTTTAGGTGAATATCTGGCGTAG-3'), which contained BamH I and Hind III sites (underlined), respectively. The PCR products were cloned into BamH I-Hind III-digested pET-28a(+) to obtain pET-28a(+)-mTaHO1 by using the $N$-terminal $6 \times$ his-tag. The integrity of the construct was verified by restriction analysis and complete DNA sequencing of the insert (GenScript, Nanjing, China). The constructed vector was transferred into Escherichia coli strain BL21 (DE3). The mTaHO1 protein expression was induced by $1 \mathrm{mM}$ isopropyl $\beta$-D-thiogalactopyranoside (IPTG) at $28{ }^{\circ} \mathrm{C}$ for $12 \mathrm{~h}$, based on the manufacturer's instructions (Novagen), and purified through Ni-NTA column and Sephadex G-25 chromatography. After centrifugation at 20,000 g in a rotor (model Avanti J-25, Beckman) for $30 \mathrm{~min}$, the purified $\mathrm{mTaHO} 1$ protein was used for the following biochemical experiments.

\subsection{HO Activity Determination}

$\mathrm{HO}$ activity was analyzed using the method described in our previous reports $[14,21,35]$. In the HO activity test, the concentration of biliverdin IX (BV) was estimated using a molar absorption coefficient at $650 \mathrm{~nm}$ of $6.25 \mathrm{mM}^{-1} \cdot \mathrm{cm}^{-1}$ in $0.1 \mathrm{M}$ HEPES-NaOH buffer (pH 7.2). One unit of activity (U) was calculated by taking the quantity of enzyme to produce $1 \mathrm{nmol}$ BV per $30 \mathrm{~min}$. Protein concentration was determined according to [36] using bovine serum albumin as the standard. $K_{\mathrm{m}}$ and $V_{\max }$ values were calculated for mTaHO1 using Lineweaver-Burk plot. For hemin, the data were obtained using the standard assay, with the concentrations varied between 1 and $20 \mu \mathrm{M}$. The effects of temperature and $\mathrm{pH}$ were determined using the standard assay conditions as described above.

\subsection{Subcellular Localization of TaHO1}

The coding region for the putative transit peptide for TaHO1 was amplified from wheat cDNA with the following primer pairs P3 (F: 5'-GCCGACTAGTCCGCGACCATCCTC-3' and R: 5'-CTCGGTACCCGGGGATCCTCTAGA-3') containing Spe I and Nco I sites (underlined), respectively, and was cloned into Spe I-Nco I-digested pCAMBIA 1302 to generate an in-frame fusion with the green fluorescent protein (GFP) reporter gene under the control of CaMV 35S promoter. The transient assays of fluorescent protein expression in Nicotiana benthamiana were performed according to [37] by using Agrobacterium tumefaciens EHA105. Preparation of bacterium and tobacco seedlings, and tobacco inoculation were carried out as described by [38]. After $2 \mathrm{~d}$ of co-cultivation, the signals 
for GFP and chloroplast autofluorescence were examined under a TCS-SP2 confocal laser scanning microscope (Leica Lasertechnik GmbH, Heidelberg, Germany).

\subsection{Gel Blot Analysis for TaHOI}

Primary antibody used was rabbit polyclonal antibody raised against recombinant mTaHO1 with a molecular mass of $31 \mathrm{kD}$. Sixty $\mu \mathrm{g}$ of protein from crude membrane fraction was subjected to SDS-PAGE using a $12.5 \%$ acrylamide resolving gel [21]. Separated proteins were then transferred to polyvinylidene difluoride (PVDF) membranes, and non-specific binding of antibodies was blocked with 5\% non-fat dried milk in phosphate-buffered saline (PBS, $\mathrm{pH} 7.4$ ) for $2 \mathrm{~h}$ at room temperature. Membranes were then incubated overnight at $4{ }^{\circ} \mathrm{C}$ with primary antibodies diluted 1:2000 in PBS buffer plus $1 \%$ non-fat dried milk. Immune complexes were detected using horseradish peroxidase (HRP)-conjugated goat anti-rabbit immunoglobulin G. The color was developed with a solution containing 3,3'-diaminobenzidine tetrahydrochloride (DAB) as the HRP substrate. Coomassie Brilliant Blue-stained gel was present to show that equal amounts of protein was loaded. Additionally, the films were scanned (Uniscan B700 ${ }^{+}$, Tsinghua Unigroup Ltd., Beijing, China) and analysed using Quantity One v4.4.0 software (Bio-Rad, USA).

\subsection{Real-Time RT-PCR Analysis}

Real-time quantification RT-PCR reactions were performed in a Mastercycler ${ }^{\circledR}$ ep realplex real-time PCR system (Eppendorf, Hamburg, Germany) using the $\mathrm{SYBR}^{\circledR}$ Premix Ex Taq ${ }^{\mathrm{TM}}$ (TaKaRa Bio Inc., Dalian, China) according to the manufacturer's instructions. The PCR reaction was performed using the following primers: for TaHOl (GenBank ID: HM014348), P4 (F: 5'-AATACTGGGTTGGAGAGA-3' and R: 5'-AGAAGTGGCAAATAAATG-3'); and for actin (GenBank ID: AB181991), P5 (F: 5'-TCTGGTGATGGTGTGAGC-3' and R: 5'-CGGTTGTTGTGAGGGAGT-3'). Gene-specific primers were designed with the software tool Primer Express (Applied Biosystems, Foster city, CA, USA). All reactions were set up in triplicate. Relative expression levels are presented as values relative to corresponding control sample at the indicated times, after normalization to actin transcript levels.

\section{Acknowledgements}

This work was supported by the National Natural Science Foundation of China (grant No. 30671248), the Priority Academic Program Development of Jiangsu Higher Education Institutions, the Education Department of Jiangsu (grant No. 200910), the Technology Support Program in Jiangsu Province, China (grant No. BE2010382), and the Fundamental Research Funds for the Central Universities (grant No. KYZ200905).

\section{References}

1. Apel, K.; Hirt, H. Reactive oxygen species: Metabolism, oxidative stress, and signal transduction. Annu. Rev. Plant Biol. 2004, 55, 373-399.

2. Salin, M.L. Toxic oxygen species and protective systems of the chloroplast. Physiol. Plant 1988, $72,681-689$. 
3. Shekhawat, G.S., Verma, K. Haem oxygenase (HO): An overlooked enzyme of plant metabolism and defence. J. Exp. Bot. 2010, 61, 2255-2270.

4. Stocker, R.; Yamamoto, Y.; McDonagh, A.F.; Glazer, A.N.; Ames, B.N. Bilirubin is an antioxidant of possible physiological importance. Science 1987, 235, 1043-1046.

5. Maines, M.D. Heme oxygenase: Function, multiplicity, regulatory mechanisms, and clinical applications. FASEB J. 1988, 2, 2557-2568.

6. Ewing, J.F.; Haber, S.N.; Maines, M.D. Normal and heat-induced patterns of expression of heme oxygenase-1 (HSP32) in rat brain: Hyperthermia causes rapid induction of mRNA and protein. J. Neurochem. 1992, 58, 1140-1149.

7. Yamaguchi, T.; Terakado, M.; Horio, F.; Aoki, K.; Tanaka, M.; Nakajima, H. Role of bilirubin as an antioxidant in an ischemia-reperfusion of rat liver and induction of heme oxygenase. Biochem. Biophys. Res. Commun. 1996, 223, 129-135.

8. Llesuy, S.F.; Tomaro, M.L. Heme oxygenase and oxidative stress. Evidence of involvement of bilirubin as physiological protector against oxidative damage. Biochim. Biophys. Acta. 1994, 1223, 9-14.

9. Platt, J.L.; Nath, K.A. Heme oxygenase: protective gene or Trojan horse. Nat. Med. 1998, 4, $1364-1365$.

10. Emborg, T.J.; Walker, J.M.; Noh, B.; Vierstra, R.D. Multiple heme oxygenase family members contribute to the biosynthesis of the phytochrome chromophore in Arabidopsis. Plant Physiol. 2006, 140, 856-868.

11. Gisk, B.; Yasui, Y.; Kohchi, T.; Frankenberg-Dinkel, N. Characterization of the haem oxygenase protein family in Arabidopsis thaliana reveals a diversity of functions. Biochem. J. 2010, 425, 425-434.

12. Fu, G.Q.; Xu, S.; Xie, Y.J.; Han, B.; Nie, L.; Shen, W.B.; Wang, R. Molecular cloning, characterization, and expression of an alfalfa (Medicago sativa L.) heme oxygenase-1 gene, MsHO1, which is pro-oxidants-regulated. Plant Physiol. Biochem. 2011, 49, 792-799.

13. Noriega, G.O.; Yannarelli, G.G.; Balestrasse, K.B.; Batlle, A.; Tomaro, M.L. The effect of nitric oxide on heme oxygenase gene expression in soybean leaves. Planta 2007, 226, 1155-1163.

14. Han, Y.; Zhang, J.; Chen, X.; Gao, Z.Z.; Xuan, W.; Xu, S.; Ding, X.; Shen, W.B. Carbon monoxide alleviates cadmium-induced oxidative damage by modulating glutathione metabolism in the roots of Medicago sativa. New Phytol. 2008, 177, 155-166.

15. Cui, W.T.; Fu, G.Q.; Wu, H.H.; Shen, W.B. Cadmium-induced heme oxygenase-1 gene expression is associated with the depletion of glutathione in the roots of Medicago sativa. Biometals 2011, 24, 93-103.

16. Yannarelli, G.G.; Noriega, G.O.; Batlle, A.; Tomaro, M.L. Heme oxygenase up-regulation in ultraviolet-B irradiated soybean plants involves reactive oxygen species. Planta 2006, 224, 1154-1162.

17. Xie, Y.J.; Ling, T.F.; Han, Y.; Liu, K.L.; Zheng, Q.S.; Huang, L.Q.; Yuan, X.X.; He, Z.Y.; $\mathrm{Hu}$, B.; Fang, L.; et al. Carbon monoxide enhances salt tolerance by nitric oxide-mediated maintenance of ion homeostasis and up-regulation of antioxidant defense in wheat seedling roots. Plant Cell Environ. 2008, 31, 1864-1881. 
18. Xie, Y.J.; Xu, S.; Han, B.; Wu, M.; Yuan, X.; Han, Y.; Gu, Q.; Xu, D.K.; Yang, Q.; Shen, W.B. Evidence of Arabidopsis salt acclimation induced by up-regulation of $H Y 1$ and the regulatory role of RbohD-derived reactive oxygen species synthesis. Plant J. 2011, 66, 280-292.

19. Xie, Y.J.; Cui, W.T.; Yuan, X.X.; Shen, W.B.; Yang, Q. Haem oxygenase-1 is associated with wheat salinity acclimation by modulating reactive oxygen species homeostasis. J. Integr. Plant Biol. 2011, 53, 653-670.

20. Chen, X.X.; Ding, X.; Xu, S.; Wang, R.; Xuan, W.; Cao, Z.Y.; Chen, J.; Wu, H.H.; Ye, M.B.; Shen, W.B. Endogenous hydrogen peroxide plays a positive role in the upregulation of heme oxygenase and acclimation to oxidative stress in wheat seedling leaves. J. Integr. Plant Biol. 2009, 51, 951-960.

21. Xuan, W.; Zhu, F.Y.; Xu, S.; Huang, B.K.; Ling, T.F.; Qi, J.Y.; Ye, M.B.; Shen, W.B. The heme oxygenase/carbon monoxide system is involved in the auxin-induced cucumber adventitious rooting process. Plant Physiol. 2008, 148, 881-893.

22. Muramoto, T.; Kohchi, T.; Yokota, A.; Hwang, I.; Goodman, H.M. The Arabidopsis photomorphogenic mutant hyl is deficient in phytochrome chromophore biosynthesis as a result of a mutation in a plastid heme oxygenase. Plant Cell 1999, 11, 335-348.

23. Izawa, T.; Oikawa, T.; Tokutomi, S.; Okuno, K.; Shimamoto, K. Phytochromes confer the photoperiodic control of flowering in rice (a short-day plant). Plant J. 2000, 22, 391-399.

24. Jin, Q.J.; Yuan, X.X.; Cui, W.T.; Han, B.; Feng, J.F.; Xu, S.; Shen, W.B. Isolation and characterization of a heme oxygenase-1 gene from Chinese cabbage. Mol. Biotechnol. 2011, doi:10.1007/s12033-011-9407-5.

25. Matera, K.M.; Zhou, H.; Migita, C.T.; Hobert, S.E.; Ishikawa, K.; Katakura, K.; Maeshima, H.; Yoshida, T.; Ikeda-Saito, M. Histidine-132 does not stabilize a distal water ligand and is not an important residue for the enzyme activity in heme oxygenase-1. Biochemistry 1997, 36, 4909-4915.

26. Sun, J.; Loehr, T.M.; Wilks, A.; Ortiz, de Montellano, P.R. Identification of histidine 25 as the heme ligand in human liver heme oxygenase. Biochemistry 1994, 33, 13734-13740.

27. Yoshida, T.; Kikuchi, G. Purification and properties of heme oxygenase from rat liver microsomes. J. Biol. Chem. 1979, 254, 4487-4491.

28. Muramoto, T.; Tsurui, N.; Terry, M.J.; Yokota, A.; Kohchi, T. Expression and biochemical properties of a ferredoxin-dependent heme oxygenase required for phytochrome chromophore synthesis. Plant Physiol. 2002, 130, 1958-1966.

29. Wilks, A.; Black, S.M.; Miller, W.L.; Ortiz de Montellano, P.R. Expression and characterization of truncated human heme oxygenase (hHO-1) and a fusion protein of hHO-1 with human cytochrome P450 reductase. Biochemistry 1995, 34, 4421-4427.

30. Liu, Y.H.; Xu, S.; Ling, T.F.; Xu, L.L.; Shen, W.B. Heme oxygenase/carbon monoxide system participates in regulating wheat seed germination under osmotic stress involving the nitric oxide pathway. J. Plant Physiol. 2010, 167, 1371-1379.

31. Besson-Bard, A.; Pugin, A.; Wendehenne, D. New insights into nitric oxide signaling in plants. Annu. Rev. Plant Biol. 2008, 59, 21-39.

32. Zimmermann, P.; Hirsch-Hoffmann, M.; Hennig, L.; Gruissem, W. GENEVESTIGATOR. Arabidopsis microarray database and analysis toolbox. Plant Physiol. 2004, 136, 2621-2632. 
33. Zilli, C.G.; Balestrasse, K.B.; Yannarelli, G.G.; Polizio, A.H.; Santa-Cruz, D.M.; Tomaro, M.L. Heme oxygenase up-regulation under salt stress protects nitrogen metabolism in nodules of soybean plants. Environ. Exp. Bot. 2008, 64, 83-89.

34. NCBI BLAST program. Available online: http://www.ncbi.nlm.nih.gov/blast (accessed on 14 September 2009).

35. Liu, K.L.; Xu, S.; Xuan, W.; Ling, T.F.; Cao, Z.Y.; Huang, B.K.; Sun, Y.G.; Fang, L.; Liu, Z.Y.; Zhao, N.; et al. Carbon monoxide counteracts the inhibition of seed germination and alleviates oxidative damage caused by salt stress in Oryza sativa. Plant Sci. 2007, 172, 544-555.

36. Bradford, M.M. A rapid and sensitive method for the quantitation of microgram quantities of protein utilizing the principle of protein-dye binding. Anal. Biochem. 1976, 72, 248-254.

37. Voinnet, O.; Rivas, S.; Mestre, P.; Baulcombe, D. An enhanced transient expression system in plants based on suppression of gene silencing by the p19 protein of tomato bushy stunt virus. Plant J. 2003, 33, 949-956.

38. Brandizzi, F.; Frangne, N.; Marc-Martin, S.; Hawes, C.; Neuhaus, J.M.; Paris, N. The destination for single-pass membrane proteins is influenced markedly by the length of the hydrophobic domain. Plant Cell 2002, 14, 1077-1092.

(C) 2011 by the authors; licensee MDPI, Basel, Switzerland. This article is an open access article distributed under the terms and conditions of the Creative Commons Attribution license (http://creativecommons.org/licenses/by/3.0/). 